\title{
Stochastic Analysis of Capillary Condensation in Disordered Mesopores
}

Cedric J. Gommes ${ }^{1}$, Anthony P. Roberts ${ }^{2}$

${ }^{1}$ Department of Chemical Engineering, University of Liège, Liège, Belgium

${ }^{2}$ School of Mathematics and Physics, University of Queensland, Brisbane, Australia

Most mesoporous materials of practical interest are disordered, which has a significant impact on capillary condensation and evaporation of vapours in their pores. The effect of disorder is often analyzed in a perturbative approach whereby elements of disorder (constriction, corrugation) are added to geometrically ideal pores. This approach has notably shown that defects can play the role of nucleation sites, and destabilise states that would otherwise be metastable. Although these results are informative for analyzing capillary condensation in ordered mesoporous materials it is unclear that they are relevant for realistically disordered materials. We propose an alternative approach [1], which consists in using a stochastic geometrical model to describe both the porous material and the condensate within the pores. This is done through a multiphase generalisation of the standard Gaussian random field model of disordered materials. The model parameters characterising the condensate provide a low-dimensional approximation of its configuration space, and we use a Derjaguin-Broekhoff-de Boer approximation to calculate the free-energy landscape. Our analysis notably questions the existence of vapour-like metastable states in realistically disordered mesoporous materials.
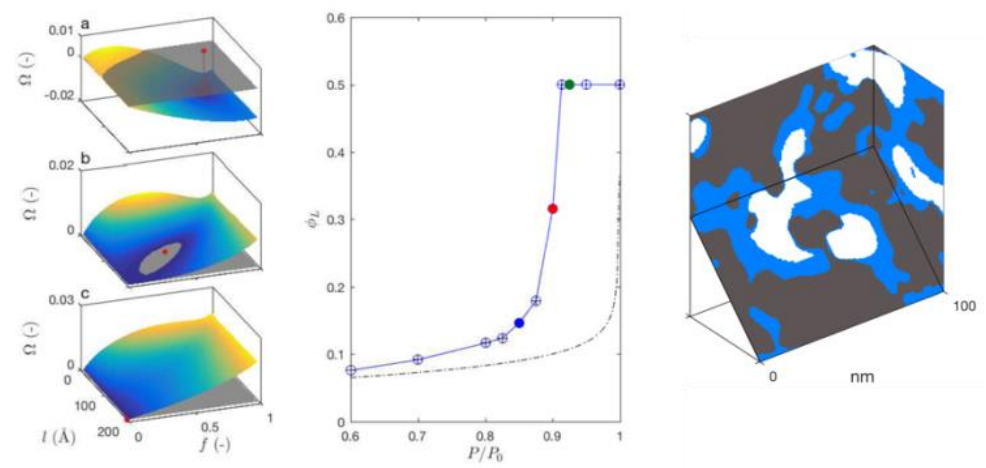

Figure 1. Left: free energy landscapes of the condensate in a mesoporous solid at $\mathrm{P} / \mathrm{P}_{0}=0.85$ (a), 0.9 (b) and 0.925 (c); Middle: corresponding equilibrium adsorption isotherm; Right: realization of the model at $\mathrm{P} / \mathrm{P}_{0}=0.9$, i.e. at the brink of capillary condensation.

[2] C. J. Gommes \& A. P. Roberts, Phys. Chem. Chem. Phys. 2018, in press, DOI: $10.1039 / \mathrm{C} 8 \mathrm{CP} 01628 \mathrm{C}$ 